\title{
Outcomes of an inpatient refeeding protocol in youth with anorexia nervosa: Rady Children's Hospital San Diego/ University of California, San Diego
}

\author{
Tamara R. Maginot ${ }^{1 *}$, Maya M. Kumar ${ }^{2}$, Jacqueline Shiels ${ }^{1}$, Walter Kaye ${ }^{1}$ and Kyung E. Rhee ${ }^{2,3}$
}

\begin{abstract}
Background: Current guidelines for nutritional rehabilitation in hospitalized restrictive eating disorder patients recommend a cautious approach to refeeding. Several studies suggest that higher calorie diets may be safe and effective, but have traditionally excluded severely malnourished patients. The goal of this study was to evaluate the safety of a higher calorie nutritional rehabilitation protocol (NRP) in a broad sample of inpatients with restrictive eating disorders, including those who were severely malnourished.

Methods: A retrospective chart review was conducted among eating disorder inpatients between January 2015 and March 2016. Patients were started on a lower calorie diet ( $\leq 1500 \mathrm{kcals} /$ day) or higher calorie diet $(\geq 1500 \mathrm{kcals} /$ day). Calorie prescription on admission was based on physician clinical judgement. The sample included patients aged 8-20 years with any DSM-5 restrictive eating disorder. Those who were severely malnourished $(<75 \%$ expected body weight [EBW]) or required tube feeding during admission were included. Multivariable regression models were used to determine whether level of nutritional rehabilitation was associated with hypophosphatemia, hypomagnesemia, or hypokalemia.
\end{abstract}

Results: The sample included 87 patients; mean age was 14.4 years (S.D. 32.7); $29 \%$ were $<75 \%$ EBW. The majority (75.8\%) was started on higher calorie diets (mean $1781 \mathrm{kcal} /$ day). Controlling for rate of calorie change, initial \%EBW, age, race/ethnicity, insurance, diagnosis, and NG/NJ tube placement, higher calorie diets were not associated with hypophosphatemia, hypomagnesemia, or hypokalemia on admission or within the first $72 \mathrm{~h}$. Increased risk of hypophosphatemia on admission was associated with lower baseline \%EBW.

Conclusion: A higher calorie NRP was tolerated in this broad population of inpatients with restrictive eating disorders. Lower \%EBW on admission was a more important predictor of hypophosphatemia than initial calorie level. Larger studies are required to demonstrate the safety of higher calorie diets in severely malnourished patients.

Keywords: Nutritional rehabilitation, Refeeding syndrome, Hypophosphatemia, Hypomagnesemia, Hypokalemia, Medical stabilization, Eating disorders, Anorexia nervosa, Avoidant Restrictive Food Intake Disorder

\footnotetext{
*Correspondence: tmaginot@ucsd.edu

${ }^{1}$ Department of Psychiatry, University of California, San Diego School of

Medicine, 4510 Executive Drive, Suite 315, San Diego, CA 92121, USA

Full list of author information is available at the end of the article
}

(c) The Author(s). 2017 Open Access This article is distributed under the terms of the Creative Commons Attribution 4.0 International License (http://creativecommons.org/licenses/by/4.0/, which permits unrestricted use, distribution, and reproduction in any medium, provided you give appropriate credit to the original author(s) and the source, provide a link to the Creative Commons license, and indicate if changes were made. The Creative Commons Public Domain Dedication waiver (http://creativecommons.org/publicdomain/zero/1.0/) applies to the data made available in this article, unless otherwise stated. 


\section{Plain english summary}

Refeeding syndrome is a concern for patients with eating disorders who are undergoing nutritional rehabilitation. It is traditionally recommended to start at a lower calorie level and advance slowly as the safest means of treatment. However, in this sample of 87 patients with restrictive eating disorders hospitalized with medical complications of malnutrition at Rady Children's Hospital, San Diego/ University of California San Diego, starting at a higher calorie level was not associated with an increased risk of electrolyte abnormalities. However, patients presenting at $<75 \%$ of expected body weight were at increased risk of developing hypophosphatemia regardless of initial calorie level. Further research is needed to determine if this method of calorie advancement is truly safe in patients with severe malnutrition (i.e., those $<75 \%$ of expected body weight).

\section{Background}

In managing patients with moderate to severe malnutrition secondary to restrictive eating disorders, the optimal rate of nutritional rehabilitation remains a subject of debate. Guidelines by the American Psychiatric Association [1] and Academy of Nutrition and Dietetics [2] currently recommend a conservative approach to nutritional rehabilitation to prevent refeeding syndrome. Refeeding syndrome occurs when malnourished patients transition to using dietary carbohydrates rather than stored macronutrients as their primary source of energy [3-5]. As the body shifts from a chronically catabolic state to an anabolic state, low body stores of phosphorus, magnesium, and potassium, in conjunction with intracellular shifting of these electrolytes (a consequence of an exaggerated insulin response) leads to low serum electrolyte levels [3-5]. Clinical sequelae may be severe, including muscle weakness and cramping, cardiac arrhythmias, vomiting, seizures, delirium, and death [3-6]. Low calorie diets have traditionally been recommended to prevent this complication during weight restoration [1, 2]. However, this approach to refeeding increases the risk of providing inadequate nutrition to cover baseline energy expenditure and facilitate weight restoration $[7,8]$. This may result in slow weight gain or even weight loss during the refeeding process, leading to prolonged medical complications related to malnutrition (e.g., bradycardia) and longer hospitalizations [7-10].

Recent research has examined whether a less conservative approach to nutritional rehabilitation can be safely performed, particularly in those with restrictive eating disorders [7-19]. Several groups have evaluated the effect of starting on a higher calorie diet (ranging from 1400 to $2400 \mathrm{kcals}$ ) among patients aged 10 to 21 years old and report shorter hospital stays [11, 13], faster weight gain [12-14], a low rate of developing hypophosphatemia during nutritional rehabilitation, and no incidence of clinical refeeding syndrome [8-15]. While rate of calorie advancement varied among groups, increasing $250 \mathrm{kcals}$ on day 2 and 3, and then by $250 \mathrm{kcals}$ every other day until day 7 , was not associated with increased risk of electrolyte abnormalities and resulted in faster weight gain [14]. Many of these groups started patients on prophylactic phosphorus supplementation which may have helped prevent the development of electrolyte abnormalities or clinical refeeding syndrome $[10,12,13]$. The use of NG tubes for nutritional rehabilitation was also not associated with adverse outcomes $[12,16,17]$. Overall, these studies suggest that starting at a higher calorie level and advancing quickly may be considered in moderately malnourished $(75-85 \%$ of expected body weight) adolescents and young adults with AN.

Unfortunately, there is limited literature on the safety of refeeding at higher calorie levels in severely malnourished patients with eating disorders. Golden et al. [11] examined a subgroup of 49 severely malnourished adolescents with anorexia nervosa (age 11-16 years) presenting at $<70 \% \mathrm{mBMI}$. Within this group, prescribed calorie level did not change the risk of electrolyte abnormalities associated with refeeding syndrome [11]. Studies have concluded that percent body weight on admissions was more associated with the development of hypophosphatemia than initial calorie prescription [9-11]. Two small descriptive studies examining patients presenting with a $\mathrm{BMI}<12$ (ranging in age from 11 to $>40$ years old) found that these patients could be safely refed with $<1200 \mathrm{kcal}$ per day, but did not assess the safety of a higher calorie diet $[16,17]$. A recent systematic review concluded that there is currently insufficient evidence to support using higher calorie prescriptions in severely malnourished patients and more research is needed in this area to ascertain the safety of starting on a higher calorie diet [18].

The primary goal of this study is to describe the nutritional rehabilitation protocol implemented at the University of California, San Diego (UCSD)/Rady Children's Hospital San Diego (RCHSD) Medical Behavioral Unit (MBU), and to examine whether higher calorie nutritional rehabilitation protocols (NRP) increase the risk of electrolyte abnormalities associated with refeeding syndrome among patients with restrictive eating disorders. The secondary goal of this study is to examine the risk of a higher initial calorie prescription in the subgroup of patients who were severely malnourished at presentation. This study builds upon existing literature by including a heterogeneous population of patients with restrictive eating disorders, including those with Avoidant Restrictive Food Intake Disorder (ARFID) or Other Specified Eating Disorder as defined in the Diagnostic and Statistical Manual, fifth edition (DSM 5) [19] and those who require NG tube feeding. 


\section{Methods}

\section{Study design and subjects}

This is an observational study of current practices on the Medical Behavioral Unit (MBU) at Rady Children's Hospital San Diego (RCHSD)/UCSD. A retrospective chart review was conducted among patients admitted between January 2015 and March 2016. RCHSD is the only children's hospital in San Diego County, serving several neighboring counties in Southern California. The MBU is an inpatient unit at RCHSD with 10 medicalsurgical beds for patients with medical complications of malnutrition.

Patients were included if they were diagnosed with AN, Other Specified Eating Disorder or Avoidant Restrictive Food Intake Disorder (ARFID) based on the Diagnostic and Statistical Manual of Mental Disorders, Fifth Edition (DSM-5) [19], and met medical criteria for hospitalization. Hospitalization criteria were based on those recommended by the Society for Adolescent Health and Medicine [8] and include: weight $<75 \%$ of the expected body weight $(\mathrm{EBW})$; resting heart rate $(\mathrm{HR})<45$ beats per minute $(\mathrm{bpm})$ while asleep or $<50 \mathrm{bpm}$ while awake; hypotension $(<90 / 45 \mathrm{mmHg})$; body temperature $<35.6^{\circ}$ Celsius; orthostatic change in systolic blood pressure $(\mathrm{SBP})>20 \mathrm{mmHg}$ or diastolic blood pressure $(\mathrm{DBP})>$ $10 \mathrm{mmHg}$; orthostatic change in HR > 30 bpm; syncope, arrhythmia, heart failure, or symptomatic pericardial effusion related to malnutrition or refeeding; dehydration; electrolyte disturbance related to malnutrition, refeeding, vomiting, or laxative/diuretic abuse; or acute food refusal necessitating tube-feeding or non-enteric feeding.

Patients were between the ages of 8 and 20 years old and were included in the analysis if this was their first or second admission to the (MBU), with no previous admission to the MBU in the preceding 30 days. Patients were excluded if they had a diagnosis of bulimia nervosa $(n=1)$, left the hospital against medical advice $(n=3)$, or were transferred to another medical or psychiatric facility $(n=2)$. Human subjects approval was obtained from the UCSD Human Research Protections Program Institutional Review Board.

\section{Nutritional rehabilitation protocol}

Upon admission, patients were started on an oral nutritional rehabilitation diet; the admitting physician relied on clinical judgment to determine the initial calorie level based on the patient's recent dietary history. Initial caloric level typically ranged from 1500 to $1800 \mathrm{kcal} /$ day, but lower calorie diets (typically $1200 \mathrm{kcal} /$ day) were used if extreme dietary restriction was reported (e.g., $<500 \mathrm{kcal} /$ day for several weeks). Daily caloric intake was titrated to achieve 150-300 g of weight gain per day, and an overall goal of 1$2 \mathrm{~kg}$ of weight gain per week. If the daily expectation for weight gain was not met for 2 days in a row, caloric intake was increased. Caloric intake was also advanced if the patient had persistent or severe cardiac complications (e.g., heart rate $<40 \mathrm{bpm}$ overnight, symptomatic postural changes in HR or BP, arrhythmia) despite meeting daily weight restoration goals. When increased, daily caloric intake was advanced in increments of $300 \mathrm{kcal} /$ day.

Nutritional rehabilitation was provided orally as three meals per day and up to three snacks per day. The MBU oral diet consisted of a 7-day rotating menu. Macronutrient content of the diet was approximately $31-36 \%$ fat, $14-19 \%$ protein, and $50 \%$ carbohydrate. All patients were required to eat in a group setting, supervised by a licensed psychiatry technician or vocational nurse, and were observed for at least 30 min after each meal or snack. Patients who refused to eat the provided food were offered the caloric equivalent as a liquid nutritional supplement Boost $^{\mathrm{TM}}$ or Nutren ${ }^{\mathrm{TM}}$, either the $1.0 \mathrm{kcal} / \mathrm{mL}$ or $1.5 \mathrm{kcal} / \mathrm{mL}$ concentration), which was comparable in macronutrient composition to the oral diet.

If the patient had difficulty eating or taking liquid supplement by mouth within the specified time limit, a nasogastric (NG) or nasojejunal (NJ) tube was inserted to provide nutrition. The NG/NJ tube was left in place and used to supplement oral intake until the patient consumed $100 \%$ of daily nutritional and fluid requirements by mouth for at least $24 \mathrm{~h}$. Decisions about calorie advancement for patients receiving tube feeds were made using identical criteria as for those who were consuming all nutrition orally (see above). Therefore, patients receiving some NG/NJ feeds during their admission were included in this analysis.

Intravenous fluids were only used in dehydrated patients who could not tolerate oral fluid replacement. A multivitamin was routinely recommended by the dietitian and additional micronutrient supplements (e.g., Vitamin D, Vitamin B12, zinc, or folate) were provided as needed.

Refeeding labs were monitored at least once daily. Patients did not receive routine prophylaxis against refeeding syndrome. Electrolyte supplementation was only initiated when decreases in serum electrolytes were identified. There was no standard protocol defining when patients should be started on electrolyte supplementation; the attending physician used clinical judgment and often started electrolyte supplementation prior to serum levels falling into the abnormal range if levels were noted to be dropping rapidly.

All patients were routinely assessed and followed by psychologists, who provided family, group, and individual therapy. Psychiatry assessments were requested based on the patient's clinical condition.

\section{Measures}

On admission, all patients were screened with a complete blood count and differential, complete metabolic panel, 
serum magnesium, serum phosphorus, prealbumin, thyroid function tests, serum zinc, serum Vitamin D-25-OH, C-reactive protein, erythrocyte sedimentation rate, serum estradiol or testosterone, urinalysis, and electrocardiogram. Subsequently, daily refeeding labs were assessed including a basic metabolic panel, serum magnesium, serum phosphorus, and urinalysis. Lowest overnight HR was obtained from continuous cardiac monitoring overnight, performed for all patients throughout their stay. Orthostatic BP and HR were obtained first thing in the morning throughout the admission. Weights were also obtained daily throughout the admission after the first morning void, wearing only a hospital gown. Height was obtained on day 1 of admission.

Overall change in calories was determined by subtracting the initial calorie level from the discharge calorie level. The rate of change in calories was calculated as overall change in calories divided by the total length of hospital stay in days. Change in lowest overnight HR was also calculated in a similar manner (lowest overnight HR the night prior to discharge - lowest overnight HR in the first $48 \mathrm{~h}$ of admission).

Expected body weight (EBW) was determined using several clinical factors. While some groups conventionally use the 50th percentile BMI to calculate expected body weight for children and adolescents [20], our program believes in customizing EBW to return each child or adolescent to the growth trajectory in which they were previously healthy. If a premorbid growth curve was available, EBW was selected to restore the patient back to the patient's premorbid BMI percentile (25th, 50th, or 75 th percentile BMI). If the patient's premorbid BMI was $>85$ th percentile but the patient was otherwise healthy, initial EBW was selected to restore the patient to a BMI at the 75th percentile. If premorbid growth patterns were unknown, the 50th percentile BMI for age and sex was used to determine EBW. Since height was only measured on day 1 , changes in BMI during the hospital stay essentially reflected changes in weight. Percent of expected body weight (\%EBW) was calculated as weight divided by EBW. Change in \%EBW was determined by subtracting the admission \%EBW from the discharge \%EBW. Rate of change in \%EBW was calculated as the overall change in \%EBW divided by the total length of stay. Severe malnutrition was defined as $\mathrm{a} \leq 75 \% \mathrm{EBW}$ and moderate malnutrition was defined at $75-85 \% \mathrm{EBW}$.

Electrolyte abnormalities were defined as a low phosphorus, magnesium, or potassium within the first $72 \mathrm{~h}$ of admission. In the current analysis, hypophosphatemia was defined as serum phosphorus $<3.0 \mathrm{mg} / \mathrm{dL}$, hypomagnesemia was defined as serum magnesium $<1.7 \mathrm{mg} / \mathrm{dL}$, and hypokalemia was defined as serum potassium $<3.3 \mathrm{mmol} / \mathrm{L}$ [11].

\section{Analysis}

Means and frequencies, $t$-tests, and chi-square tests were used to describe the sample and compare those who were started on a lower calorie nutritional rehabilitation diet ( $\leq 1500 \mathrm{kcals} /$ day) to those who were started on a higher calorie diet $(\geq 1500 \mathrm{kcals} /$ day $)$. The aim of this study was to examine whether starting on a higher or lower calorie nutritional rehabilitation diet affected overall change in \%EBW, overall change in calories, rate of change in \%EBW, rate of change in calories, change in $\mathrm{HR}$, change in weight, length of hospital stay (LOS), and electrolyte abnormalities (specifically hypophosphatemia, hypomagnesemia, or hypokalemia). Multivariable general linear regression models were used to examine the relationship between overall change in \%EBW and calories, rate of change in \%EBW and calories, change in $\mathrm{HR}$ and weight, LOS, and level of initial nutritional rehabilitation (high vs. low). \%EBW on admission was included as a covariate in all models since this indicated severity of illness and may have been used by the physician to determine what calorie level to initiate. Standard demographic characteristics, diagnosis, and covariates with a $p$-value $\leq 0.05$ were also included in the models. Significance level was set at $p \leq 0.05$. Multivariable logistic regression models were used to determine whether level of nutritional rehabilitation was associated with hypophosphatemia, hypomagnesemia, or hypokalemia on the first day of admission and within the next $72 \mathrm{~h}$. \%EBW, rate of change in calories, standard demographic characteristics, diagnosis, and covariates with a $p$-value $\leq 0.05$ were included as covariates. SAS v9.4 (Cary, NC) was used for analysis.

\section{Results}

A total of 87 patients hospitalized during the study period met inclusion criteria. The mean age of patients was 14.4 years (S.D. 2.7 years) with a range of 8 to 20 years. The mean LOS was 15.3 days (S.D. 9.6 days). Patients had a \%EBW of $80.6 \%$ (S.D. 10.5\%) on admission and gained an average of $3.1 \mathrm{~kg}$ (S.D. $2.1 \mathrm{~kg}$ ) during their stay. This translates to a mean change in \%EBW of $5.41 \%$ (S.D. $4.32 \%$ ) during their stay, or $0.35 \% \mathrm{EBW} /$ day (S.D. 0.30\% EBW/day). Most patients had AN-restrictive subtype (66.7\%); $16.1 \%$ had AN-binge/purge subtype, 5.7\% were diagnosed with Other Specified Eating Disorder, and $11.5 \%$ were diagnosed with ARFID. Fifteen patients (13.8\%) required nasogastric/nasojejunal (NG/ NJ) feeds during admission. The overall rate of change in calories was $109 \mathrm{kcals} /$ day (S.D. $50.1 \mathrm{kcal} /$ day).

Baseline characteristics of patients in the low and high calorie groups are summarized in Table 1. The majority $(75.8 \%)$ of patients were started on a higher calorie diet on admission (mean $1781 \mathrm{kcal} /$ day, S.D. $315 \mathrm{kcal} /$ day, range $1500-3000 \mathrm{kcal} /$ day). The low calorie group received 
Table 1 Characteristics of patients admitted to the Medical Behavioral Unit $(n=87)$

\begin{tabular}{|c|c|c|c|c|c|c|c|}
\hline & \multicolumn{3}{|c|}{ Low Calorie $(n=21)$} & \multicolumn{3}{|c|}{ High Calorie $(n=66)$} & \multirow[b]{2}{*}{$P$-value } \\
\hline & Mean (S.D.) & Min & Max & Mean (S.D.) & Min & Max & \\
\hline Age (yrs) & $14.66(2.83)$ & 10.0 & 19.0 & $14.3(2.6)$ & 8.0 & 19.0 & 0.59 \\
\hline LOS (days) & $20.7(11.2)$ & 5.0 & 48.0 & $13.5(8.37)$ & 3.0 & 41.0 & $<0.01$ \\
\hline \%EBW - Admit & $78.7(12.7)$ & 61.5 & 118.0 & $81.25(9.8)$ & 58.8 & 103.0 & 0.34 \\
\hline \%EBW - Discharge & $85.12(11.2)$ & 71.2 & 120.0 & $85.5(8.4)$ & 60.2 & 104.0 & 0.76 \\
\hline Change in \%EBW & $6.77(4.93)$ & 0.44 & 17.2 & $4.98(4.1)$ & -4.8 & 16.4 & 0.10 \\
\hline Calories - Admit (Kcals) & $1185(65.4)$ & 1000 & 1300 & $1781(315.2)$ & 1500 & 3000 & \\
\hline Calories - Discharge (Kcals) & $3019(897.0)$ & 1400 & 4500 & $3150(784.8)$ & 1800 & 5500 & 0.52 \\
\hline \multirow[t]{2}{*}{ Change in Calories (Kcals) } & $1833(906.8)$ & 200 & 3300 & $1369(725.3)$ & 0 & 3200 & 0.02 \\
\hline & \multicolumn{3}{|l|}{ Frequency (\%) } & \multicolumn{3}{|l|}{ Frequency (\%) } & $P$-value \\
\hline Female & \multicolumn{3}{|l|}{81.0} & \multicolumn{3}{|l|}{84.9} & 0.67 \\
\hline \multicolumn{7}{|l|}{ Diagnosis: } & 0.20 \\
\hline Anorexia-restrict & \multicolumn{3}{|l|}{47.6} & \multicolumn{3}{|l|}{72.7} & \\
\hline Anorexia-binge/purge & \multicolumn{3}{|l|}{23.8} & \multicolumn{3}{|l|}{13.6} & \\
\hline Other Specified Eating Disorder & \multicolumn{3}{|l|}{9.5} & \multicolumn{3}{|l|}{4.6} & \\
\hline ARFID & \multicolumn{3}{|l|}{19.1} & \multicolumn{3}{|l|}{9.1} & \\
\hline Vegetarian history & \multicolumn{3}{|l|}{14.3} & \multicolumn{3}{|l|}{13.6} & 0.94 \\
\hline Vegan history & \multicolumn{3}{|l|}{9.5} & \multicolumn{3}{|l|}{7.6} & 0.78 \\
\hline Previously overweight/obese & \multicolumn{3}{|l|}{23.8} & \multicolumn{3}{|l|}{18.2} & 0.57 \\
\hline First time admission & \multicolumn{3}{|l|}{81.0} & \multicolumn{3}{|l|}{87.9} & 0.42 \\
\hline Insurance & & & & & & & 0.02 \\
\hline Private & \multicolumn{3}{|l|}{57.1} & \multicolumn{3}{|l|}{81.8} & \\
\hline Public & 42.9 & & & 18.2 & & & \\
\hline Race/Ethnicity: & & & & & & & 0.34 \\
\hline Caucasian & 76.2 & & & 66.7 & & & \\
\hline Latino & 9.5 & & & 13.6 & & & \\
\hline African-American & 4.8 & & & 0 & & & \\
\hline Asian & 4.8 & & & 10.6 & & & \\
\hline Mixed/Other & 4.7 & & & 9.1 & & & \\
\hline Family history: & & & & & & & \\
\hline Eating Disorder & 14.3 & & & 39.4 & & & 0.03 \\
\hline Anxiety & 23.8 & & & 28.8 & & & 0.66 \\
\hline Depression & 47.6 & & & 48.5 & & & 0.94 \\
\hline Obsessive-Compulsive disorder & 4.8 & & & 10.6 & & & 0.42 \\
\hline Drug/Alcohol abuse & 38.1 & & & 34.9 & & & 0.79 \\
\hline NG/NJ Tube & 28.6 & & & 9.1 & & & 0.02 \\
\hline
\end{tabular}

\%EBW \% of Expected Body Weight, ARFID Avoidant Restrictive Food Intake Disorder, NG/NJ Nasogastric/Nasojejeunal

a mean of $1185 \mathrm{kcal} /$ day (S.D. $65 \mathrm{kcal} /$ day, range $1000-$ $1300 \mathrm{kcal} /$ day). Patients in the low calorie group had longer LOS (20.7 days vs. 13.5 days, $p<0.01)$. They were also more likely to have an NG/NJ tube placed $(28.6 \%$ vs. $9.1 \%$, $p=0.02$ ). There was no difference in diagnosis by calorie level. There were no differences in mean laboratory values except that patients in the low calorie group had higher ALT (38.3 vs. $31.9, p=0.05$ ) (Table 2). The low calorie group also had higher overnight HR on admission (49.4 vs 43.8, $p=0.05)$. There were no differences in orthostatic vital signs or ECG findings.

In the multivariable analysis (Table 3), starting on a higher calorie diet was associated with a lower change in HR (-6.3 bpm, S.D. 2.9, $p=0.03)$, controlling for initial \%EBW, NG/NJ tube placement, and other demographic characteristics. There was no effect on absolute change 
Table 2 Vital signs and Laboratory values of patients admitted to the Medical Behavioral Unit $(n=87$ )

\begin{tabular}{|c|c|c|c|c|c|c|c|}
\hline & \multicolumn{3}{|c|}{ Low Calorie $(n=21)$} & \multicolumn{3}{|c|}{ High Calorie $(n=66)$} & \multirow[b]{2}{*}{$P$-value } \\
\hline & Mean (S.D.) & Min & Max & Mean (S.D.) & Min & Max & \\
\hline Temperature & $36.4(0.8)$ & 35.0 & 39.0 & $36.2(0.5)$ & 35.0 & 37.0 & 0.17 \\
\hline \multicolumn{8}{|l|}{ Orthostatic Vital Signs: } \\
\hline Lying Systolic Blood Pressure & $107(12)$ & 89 & 135 & $104(12)$ & 83 & 137 & 0.24 \\
\hline Lying Diastolic Blood Pressure & $67(11)$ & 49 & 88 & $61(9)$ & 80 & 63 & 0.03 \\
\hline Lying Heart Rate (bpm) & $64(24.5)$ & 40 & 121 & $57(17)$ & 31 & 116 & 0.18 \\
\hline Standing Systolic Blood Pressure & $104(13.3)$ & 88 & 131 & $101(11)$ & 72 & 128 & 0.45 \\
\hline Standing Diastolic Blood Pressure & $67(9.5)$ & 49 & 83 & $64(12)$ & 40 & 103 & 0.88 \\
\hline Standing Heart Rate (bpm) & $71(25.5)$ & 39 & 125 & $73(21)$ & 42 & 113 & 0.75 \\
\hline \multicolumn{8}{|l|}{ Labs: } \\
\hline Sodium (mmol/L) & $140.4(3.8)$ & 134.0 & 148.0 & $141.1(3.3)$ & 131.0 & 147.0 & 0.20 \\
\hline Potassium (mmol/L) & $3.8(0.9)$ & 3.0 & 7.0 & $3.6(0.6)$ & 3.0 & 5.0 & 0.27 \\
\hline Chloride (mmol/L) & $102.7(4.3)$ & 95.0 & 113.0 & $102.0(2.9)$ & 92.0 & 111.0 & 0.80 \\
\hline Bicarb (mmol/L) & 26.6 (3.6) & 18.0 & 32.0 & $27.5(2.6)$ & 20.0 & 33.0 & 0.23 \\
\hline BUN (mg/dl) & $13.2(7.2)$ & 2.0 & 31.0 & $15.8(6.9)$ & 1.0 & 37.0 & 0.14 \\
\hline Creatinine (mg/dL) & $0.1(0.03)$ & 0.00 & 1.0 & $0.03(0.17)$ & 0.0 & 1.0 & 0.21 \\
\hline Glucose (mg/dL & $87.1(30.4)$ & 54.0 & 180.0 & $83.0(17.1)$ & 31.0 & 142.0 & 0.45 \\
\hline Phosphorus (mg/dL) & $3.7(0.6)$ & 2.0 & 4.0 & $3.8(0.7)$ & 1.0 & 5.0 & 0.72 \\
\hline Magnesium (mg/dL) & $1.7(0.5)$ & 1.0 & 2.0 & $1.89(0.3)$ & 1.0 & 2.0 & 0.07 \\
\hline AST (U/L) & $34.1(13.4)$ & 10.2 & 76.0 & $35.4(12.0)$ & 10.0 & 74.0 & 0.69 \\
\hline $\mathrm{ALT}(\mathrm{U} / \mathrm{L})$ & $38.3(15.5)$ & 15.0 & 66.0 & $31.9(12.3)$ & 14.0 & 98.0 & 0.05 \\
\hline Prealbumin (mg/dL) & $19.1(6.2)$ & 10.0 & 30.0 & $20.9(4.2)$ & 11.0 & 32.0 & 0.15 \\
\hline Vitamin D (ng/mL) & $35.6(7.7)$ & 23.0 & 44.0 & $37.8(13.5)$ & 15.0 & 79.0 & 0.66 \\
\hline WBC (thousand/uL) & $5.8(1.6)$ & 3.5 & 11.0 & $5.4(1.4)$ & 1.6 & 9.7 & 0.26 \\
\hline $\mathrm{Hgb}(\mathrm{g} / \mathrm{dL})$ & $12.7(1.2)$ & 11.0 & 14.0 & $12.9(1.3)$ & 8.7 & 16.0 & 0.54 \\
\hline CRP (mg/dL) & $0.7(0.4)$ & 0.5 & 2.2 & $0.60(0.26)$ & 0.5 & 1.9 & 0.30 \\
\hline Estradiol (pg/mL) & $7.8(7.7)$ & 2.0 & 24.0 & $13.4(15.3)$ & 1.0 & 65.0 & 0.17 \\
\hline Testosterone (ng/mL) & $8.0(5.3)$ & 2.0 & 12.0 & $24.5(23.2)$ & 1.4 & 74.0 & 0.25 \\
\hline \multicolumn{8}{|l|}{ ECG: } \\
\hline QTc (ms) & $0.410(.031)$ & 0.346 & 0.457 & $0.408(.026)$ & 0.335 & 0.470 & 0.81 \\
\hline \multicolumn{8}{|l|}{ Cardiac Monitoring: } \\
\hline Lowest HR overnight - Admit (bpm) & $49.4(14.1)$ & 29.0 & 78.0 & $43.8(10.1)$ & 31.0 & 79.0 & 0.05 \\
\hline Lowest HR overnight - Discharge (bpm) & $68.8(18.7)$ & 45.0 & 105.0 & $54.7(9.5)$ & 42.0 & 79.0 & $<0.001$ \\
\hline \multirow[t]{2}{*}{ Change in HR } & $18.9(16.0)$ & -10.0 & 49.0 & $10.5(7.6)$ & -21.0 & 31.0 & $<0.01$ \\
\hline & \multicolumn{3}{|l|}{ Frequency (\%) } & \multicolumn{3}{|l|}{ Frequency (\%) } & \\
\hline Hypophosphatemia $^{a}$ & 57.1 & & & 51.5 & & & 0.65 \\
\hline Hypomagnesemia $^{a}$ & 52.4 & & & 51.5 & & & 0.94 \\
\hline Hypokalemia $^{a}$ & 19.1 & & & 4.6 & & & 0.03 \\
\hline
\end{tabular}

bpm beats per minute, HR Heart Rate

${ }^{\mathrm{a}}$ Frequency of the development of low electrolyte levels in the first $72 \mathrm{~h}$ of admission

in \%EBW or rate of change in \%EBW. While starting on a higher calorie diet was associated with lower change in calories during the admission ( -526.5 , S.D. 208.6, $p=0.01$ ), it was not associated with the rate of change in calories. Length of stay was associated with NG/NJ tube placement (7.2 days, S.D. 2.7, $p=0.009$ ) and inversely associated with \%EBW on admission (-0.4, S.D. 0.1, $p<0.001$ ). However, if children who were on NG/NJ tube feeds were removed from the analysis $(n=12)$, length of stay was significantly associated with starting on a higher 
Table 3 Relationship between starting on a high calorie nutritional rehabilitation protocol and inpatient outcomes: Change in \%EBW, Change in Calories, Rate of Change in \%EBW, Rate of Change in Calories, Length of Stay, Change in Weight, and Change in Heart Rate

\begin{tabular}{|c|c|c|c|c|c|c|c|c|}
\hline & \multicolumn{2}{|c|}{ Change in \%EBW } & \multicolumn{2}{|c|}{ Change in Calories (kcals) } & \multicolumn{2}{|c|}{ Rate of Change in \%EBW } & \multicolumn{2}{|c|}{ Rate of Change in Calories (kcals) } \\
\hline & Estimate (S.E.) & $p$-value & Estimate (S.E.) & $p$-value & Estimate (S.E.) & $p$-value & Estimate (S.E.) & $p$-value \\
\hline Higher Calorie Refeeding Diet & $-1.07(1.04)$ & 0.31 & $-526.5(208.6)$ & 0.01 & $0.01(0.08)$ & 0.88 & $-13.2(12.4)$ & 0.29 \\
\hline \%EBW on admission & $-0.22(0.04)$ & $<0.0001$ & $-12.6(8.9)$ & 0.16 & $-0.01(0.00)$ & 0.03 & $1.7(0.5)$ & 0.002 \\
\hline Age & $-0.14(0.20)$ & 0.49 & $86.8(39.9)$ & 0.03 & $-0.00(0.02)$ & 0.99 & $3.6(2.4)$ & 0.13 \\
\hline \multicolumn{9}{|l|}{ Race/Ethnicity } \\
\hline Caucasian (reference) & 0.0 & & 0.0 & & 0.0 & & 0.0 & \\
\hline Latino & $1.19(1.36)$ & 0.38 & $-47.2(272.3)$ & 0.86 & $0.11(0.10)$ & 0.29 & $4.2(16.2)$ & 0.80 \\
\hline African American & $-5.68(4.08)$ & 0.17 & $-1352.1(815.1)$ & 0.10 & $-0.28(0.32)$ & 0.38 & $-55.3(48.6)$ & 0.26 \\
\hline Asian & $3.02(1.43)$ & 0.04 & $-19.7(287.3)$ & 0.94 & $0.36(0.11)$ & 0.002 & $-23.2(17.1)$ & 0.18 \\
\hline Mixed/Other & $1.23(1.47)$ & 0.41 & $154.2(294.8)$ & 0.60 & $0.14(0.11)$ & 0.22 & $-12.3(17.6)$ & 0.49 \\
\hline Insurance Status (Public) & $2.28(1.03)$ & 0.03 & $174.6(207.0)$ & 0.40 & $0.10(0.08)$ & 0.23 & $-26.8(12.3)$ & 0.03 \\
\hline \multicolumn{9}{|l|}{ Diagnosis } \\
\hline Anorexia-restrict (reference) & 0.0 & & 0.0 & & 0.0 & & 0.0 & \\
\hline Anorexia-binge/purge & $-0.26(1.27)$ & 0.84 & $-277.8(254.9)$ & 0.28 & $-0.09(0.10)$ & 0.43 & $-30.4(15.2)$ & 0.05 \\
\hline Other Specified Eating Disorder & $0.67(1.85)$ & 0.72 & $-337.3(370.8)$ & 0.37 & $0.05(0.14)$ & 0.72 & $0.3(22.1)$ & 0.99 \\
\hline ARFID & $-1.11(1.38)$ & 0.42 & $-198.0(276.7)$ & 0.48 & $-0.07(0.11)$ & 0.52 & $-36.9(16.5)$ & 0.03 \\
\hline \multirow[t]{3}{*}{ NG/NJ tube } & $1.59(1.20)$ & 0.19 & $-171.2(240.1)$ & 0.48 & $0.01(0.09)$ & 0.89 & $-48.1(14.3)$ & 0.001 \\
\hline & \multicolumn{2}{|c|}{ Length of Stay (days) } & \multicolumn{3}{|c|}{ Change in Weight (kg) } & \multicolumn{3}{|c|}{ Change in Heart Rate (bpm) } \\
\hline & Estimate (S.E.) & $p$-value & Estimate (S.E.) & & $p$-value & Estimate ( & (S.E.) & $p$-value \\
\hline Higher Calorie Refeeding Diet & $-3.2(2.3)$ & 0.18 & $-0.52(0.51)$ & & 0.32 & $-6.3(2.9)$ & & 0.03 \\
\hline \%EBW on admission & $-0.4(0.1)$ & $<0.001$ & $-0.09(0.02)$ & & $<0.0001$ & $-0.4(0.1)$ & & 0.001 \\
\hline Age & $0.4(0.4)$ & 0.35 & $0.15(0.10)$ & & 0.12 & $-0.3(0.5)$ & & 0.53 \\
\hline \multicolumn{9}{|l|}{ Race/Ethnicity } \\
\hline Caucasian (reference) & 0.0 & & 0.0 & & & 0.0 & & \\
\hline Latino & $-0.5(3.1)$ & 0.86 & $0.66(0.67)$ & & 0.33 & $0.6(3.8)$ & & 0.87 \\
\hline African American & $6.3(9.2)$ & 0.50 & $-1.79(2.01)$ & & 0.38 & $-1.4(11.0$ & & 0.90 \\
\hline Asian & $0.6(3.2)$ & 0.85 & $0.49(0.71)$ & & 0.49 & $-5.4(3.9)$ & & 0.16 \\
\hline Mixed/Other & $0.9(3.3)$ & 0.80 & $0.42(0.73)$ & & 0.56 & $-1.1(4.0)$ & & 0.78 \\
\hline Insurance Status (Public) & $4.6(2.3)$ & 0.05 & $0.76(0.51)$ & & 0.14 & $4.1(2.8)$ & & 0.15 \\
\hline \multicolumn{9}{|l|}{ Diagnosis } \\
\hline Anorexia-restrict (reference) & 0.0 & & 0.0 & & & 0.0 & & \\
\hline Anorexia-binge/purge & $1.2(2.9)$ & 0.67 & $-0.04(0.63)$ & & 0.95 & $0.1(3.5)$ & & 0.98 \\
\hline Other Specified Eating Disorder & $-1.0(4.2)$ & 0.82 & $-0.32(0.91)$ & & 0.72 & $1.5(5.0)$ & & 0.76 \\
\hline ARFID & $0.4(3.1)$ & 0.89 & $-0.54(0.68)$ & & 0.43 & $1.0(3.8)$ & & 0.80 \\
\hline NG/NJ tube & $7.2(2.7)$ & 0.009 & $0.74(0.59)$ & & 0.22 & $0.1(3.3)$ & & 0.96 \\
\hline
\end{tabular}

Multivariable linear regression models were created to examine the relationship between starting on a high calorie nutritional rehabilitation protocol and several outcomes. All models controlled for age of patient, \% expected body weight on admission, race/ethnicity, insurance status, diagnosis, and whether an NG/NJ tube was used during the admission. Adjusted estimates with standard errors and $p$-values are shown for each model

calorie diet ( -4.67 days, S.D. 2.20, $p=0.04)$ (full model not shown in table).

Initial calorie level was not associated with the development of hypophosphatemia, hypomagnesemia, or hypokalemia on admission or in the first $72 \mathrm{~h}$, controlling for rate of calorie change, age, race/ethnicity, insurance, diagnosis, and NG/NJ tube placement (Tables 4 and 5). Rate of calorie change was also not associated with electrolyte abnormalities. However, \%EBW was associated with hypophosphatemia on admission and trended towards hypomagnesemia such that those with higher \%EBW had lower odds of developing these electrolyte abnormalities. 
Table 4 Factors associated with Electrolyte Abnormalities Associated with Refeeding Syndrome on admission

\begin{tabular}{|c|c|c|c|c|c|c|}
\hline & \multicolumn{2}{|c|}{ Hypophosphatemia } & \multicolumn{2}{|c|}{ Hypomagnesemia } & \multicolumn{2}{|l|}{ Hypokalemia } \\
\hline & OR $(95 \% \mathrm{Cl})$ & $p$-value & OR $(95 \% \mathrm{Cl})$ & $p$-value & OR $(95 \% \mathrm{Cl})$ & $p$-value \\
\hline Higher Calorie Refeeding Diet & $0.92(0.24,3.50)$ & 0.91 & $0.89(0.23,3.43)$ & 0.87 & $>100(<0.01,>100)$ & 0.38 \\
\hline \%EBW on admit & $0.94(0.88,1.00)$ & 0.05 & $0.94(0.88,1.00)$ & 0.06 & $0.04(<0.01,36.97)$ & 0.36 \\
\hline Rate of change in calories & $1.00(0.99,1.01)$ & 0.51 & $1.00(0.99,1.01)$ & 0.56 & $1.36(0.73,2.54)$ & 0.33 \\
\hline
\end{tabular}

Multivariable logistic regression models were used to determine whether level of nutritional rehabilitation on admission was associated with hypophosphatemia, hypomagnesemia, or hypokalemia on the first day of admission. All models controlled for \% expected body weight on admission, rate of change in calories, as well as age race/ethnicity, insurance status, diagnosis, and NG/NJ tube status

A secondary analysis was conducted on severely malnourished patients who were $<75 \%$ EBW on admission $(n=26)$. Starting on a higher calorie level compared to the lower calorie level was not associated with increased risk of developing hypophosphatemia ( $69 \%$ vs $60 \%, p=0.65$ ), hypomagnesemia ( $63 \%$ vs. $50 \%$, $p=0.53$ ), or hypokalemia ( $6 \%$ vs $10 \%, p=0.72$ ) in the first $72 \mathrm{~h}$. In other analyses, severely malnourished patients who were started on a lower calorie diet were more likely to have an NG/NJ tube than those who were started on a higher calorie diet ( $30 \%$ vs. $0 \%, p=0.02)$. Additionally, they were more likely to have more than one admission to the unit than those severely malnourished patients who were started on a higher calorie diet $(40 \%$ vs. $6 \%, p=0.03)$.

\section{Discussion}

This paper includes a heterogeneous sample of adolescents diagnosed with all DSM-5 restrictive eating disorders and included a subgroup of severely malnourished patients presenting at $<75 \% \mathrm{EBW}$. The study aimed to determine any clinical sequelae associated with starting on a higher calorie diet than traditionally recommended. Our results support the emerging literature suggesting that higher calorie nutritional rehabilitation can be safe and effective [7-18].

Consistent with findings from previous studies [11-13], incidence of electrolyte abnormalities in our sample was not associated with the rate of caloric advancement or initial calorie level. Rather, initial hypophosphatemia was more common among patients with a lower \%EBW on admission, suggesting that the degree of body depletion is a more important predictor of electrolyte abnormalities. Previous studies have suggested that patients presenting with a weight $<80 \%$ of median body weight are at elevated risk for electrolyte abnormalities associated with refeeding syndrome $[3,5]$. Our data suggested that with every $1 \%$ decrease in $\% \mathrm{EBW}$ on admission, the odds of hypophosphatemia increased by $6 \%$. However, among our subset of severely malnourished patients presenting at $<75 \% \mathrm{EBW}$, starting at a higher calorie diet was not associated with a higher risk of hypophosphatemia, hypomagnesemia, or hypokalemia. This subgroup was relatively small to draw definitive conclusions about the safety of higher calorie diets in this low-weight group, and additional studies with larger sample sizes are needed.

Higher calorie diets (1781 kcals, range 1500-3000 kcals) had no effect on the rate of change in \%EBW which is consistent with findings by Golden et al. [11]. Interestingly, higher calorie diets were not associated with shorter lengths of stay when NG/NJ tube fed patients were included in the analysis. Patients needing NG/N) tubes typically had significant behavioral components to their food refusal that required more prolonged behavioral intervention. They also had medical complications (e.g., superior mesenteric artery syndrome, intractable vomiting) that delayed the start of safe oral feeding, thus increasing length of stay. Therefore, the use of NG/NJ tubes appears to reflect a more severe population that needed a longer length of stay to resolve these issues. However, in post-hoc analysis removing this sub-population who received NG/NJ feeds, higher calorie diets were again associated with shorter lengths of stay ( -4.6 days, S.D. 2.2, $p=0.04$ ) which is consistent with the previous research $[11,13]$.

It is also interesting to note that in our sample of patients with a broad spectrum of disease characteristics, only half had low serum phosphorus and magnesium levels.

Table 5 Factors associated with Electrolyte Abnormalities Associated with Refeeding Syndrome in the first $72 \mathrm{~h}$ of admission

\begin{tabular}{|c|c|c|c|c|c|c|}
\hline & \multicolumn{2}{|c|}{ Hypophosphatemia } & \multicolumn{2}{|c|}{ Hypomagnesemia } & \multicolumn{2}{|l|}{ Hypokalemia } \\
\hline & OR $(95 \% \mathrm{Cl})$ & $p$-value & OR $(95 \% \mathrm{Cl})$ & $p$-value & OR $(95 \% \mathrm{Cl})$ & $p$-value \\
\hline Higher Calorie Refeeding Diet & $0.79(0.22,2.89)$ & 0.72 & $0.82(0.22,2.96)$ & 0.76 & $0.16(0.01,2.44)$ & 0.19 \\
\hline \%EBW on admit & $0.96(0.90,1.01)$ & 0.14 & $0.96(0.91,1.02)$ & 0.18 & $1.05(0.94,1.18)$ & 0.39 \\
\hline Rate of change in calories & $0.99(0.98,1.01)$ & 0.12 & $0.99(0.98,1.01)$ & 0.17 & $0.98(0.94,1.01)$ & 0.12 \\
\hline
\end{tabular}

Multivariable logistic regression models were used to determine whether level of nutritional rehabilitation on admission was associated with hypophosphatemia, hypomagnesemia, or hypokalemia within the first $72 \mathrm{~h}$ of admission. All models controlled for \% expected body weight on admission, rate of change in calories, as well as age race/ethnicity, insurance status, diagnosis, and NG/NJ tube status 
However, without supplementation, many in this group resolved their electrolyte abnormality simply with oral nutrition. This suggests that prophylactic supplementation against refeeding syndrome, even among patients receiving high calorie diets, may be unnecessary if refeeding labs are closely observed. While daily lab draws have their own potential consequences, close observation may be a viable option and could prevent the use of unnecessary medications [21].

While this study adds to the existing literature on nutritional rehabilitation by including a broader range of diagnoses and disease characteristics, there were some limitations. A larger sample size would have allowed for stratified analyses among different \%EBW groups so we could more clearly determine the risk of refeeding syndrome among patients with differing degrees of malnutrition. Additionally, physician clinical judgment played a role in initial calorie determination and calorie advancement that was difficult to quantify. This may have affected the lack of association between electrolyte abnormalities and initial calorie level, since those with low electrolyte levels could have been systematically started on lower calorie diets. However, initial calorie levels were often determined before lab results were back, thereby limiting this potential confounder. Due to the nature of selfreported data, we were also unable to accurately measure the rate of recent weight loss, which likely affects cardiovascular stability and risk of refeeding syndrome; this should be included in future analyses. Finally, we can only make limited conclusions in a descriptive or observational study, and a prospective randomized control trial is necessary for more definitive conclusions regarding the safety of higher initial calorie prescriptions.

\section{Conclusion}

The results of this study continue to support the use of higher calorie nutritional rehabilitation diets among a heterogeneous group of patients with all DSM5 restrictive eating disorders, including those who are severely malnourished. Low \%EBW on admission appears to be a more important predictor of electrolyte abnormalities than initial calorie level or rate of caloric advancement. Larger studies are required to demonstrate the safety of higher calorie diets, particularly in severely malnourished patients.

\section{Acknowledgements}

We wish to gratefully acknowledge Jesslyn Goldbaum, MA and Ceon Kang, DO for the time spent on data entry and data management. We also wish to thank the University of Minnesota Children's Hospital and Children's Hospital of Philadelphia for their collaboration on this project.

\section{Funding}

This is an unfunded study.

\section{Authors' contributions}

TRM participated in the initial development of this project, data collection, interpretation of the data, and was responsible for writing of this manuscript. MMK participated in the initial development of this project, data collection, interpretation of the data, and contributed to the writing and editing of this manuscript. JS participated in the initial development of this project, data collection, interpretation of the data, and editing of the manuscript. WK contributed to the development of this project, interpretation of the data, and editing of the manuscript. KER participated in the initial development of this project, conducted the analysis, interpreted the data, and contributed to the editing of this manuscript. All authors read and approved the final manuscript.

\section{Competing interests}

The authors declare that they have no competing interests.

\section{Consent for publication}

Not applicable.

\section{Ethics approval and consent to participate}

Human subjects approval and ethics approval was obtained from the UCSD/ RCHSD Human Research Protections Program Institutional Review Board, approval \#160902. A waiver of HIPAA authorization was granted by the UCSD/RCHSD Human Research Protections Program Institutional Review Board.

\section{Author details}

'Department of Psychiatry, University of California, San Diego School of Medicine, 4510 Executive Drive, Suite 315, San Diego, CA 92121, USA. ${ }^{2}$ Division of Academic General Pediatrics and Community Health, Department of Pediatrics, University of California, San Diego School of Medicine and Rady Children's Hospital of San Diego, 7910 Frost Street, Suite 300, San Diego, CA 92123, USA. ${ }^{3} 9500$ Gilman Drive, MC 0874, La Jolla, CA 92093, USA.

Received: 25 August 2016 Accepted: 14 December 2016

Published online: 03 January 2017

\section{References}

1. Yager J, Devlin MJ, Halmi KA, Herzog DB, Michell JE, Powers P, Zerbe KJ. Treatment of patients with eating disorders, third edition. American Psychiatric Association. Am J Psychiatry. 2006;163(7 Suppl):4-54.

2. American Dietetic Association. Position of the American Dietetic Association: Nutrition intervention in the treatment of anorexia nervosa, bulimia nervosa, and other eating disorders. J Am Diet Assoc. 2006;106: 2073-82.

3. Dunn RL, Stettler N, Mascarenhas MR. Refeeding syndrome in hospitalized pediatric patients. Nutr Clin Pract. 2003;18:327-32.

4. Fuentebella J, Kerner JA. Refeeding Syndrome. Pediatr Clin N Am. 2009;56: 1201-10.

5. Solomon SM, Kirby DF. The refeeding syndrome: a review. J Parenter Enter Nutr. 1990;14:90-7.

6. Norris ML, Pinhas $L$, Nadeau PO, et al. Delirium and refeeding syndrome in anorexia nervosa. Int J Eat Disord. 2012;45:429-42.

7. Katzman DK. Refeeding hospitalized adolescents with anorexia nervosa: Is "start low, advance slow" urban legend or evidence based? J Adolesc Health. 2012;50:1-2.

8. Golden NH, Katzman DK, Sawyer SM, et al. Medical Management of Restrictive Eating Disorders in Adolescents and Young Adults. Position Paper of the Society for Adolescent Health and Medicine. J Adolesc Health. 2015;53:121-5.

9. Redgrave GW, Coughlin JW, Schreyer CC, et al. Refeeding and Weight Restoration Outcomes in Anorexia Nervosa: Challenging Current Guidelines. Int J Eat Disord. 2015;48:866-73.

10. Whitelaw M, Gilbertson H, Lam P-Y, Sawyer S. Does aggressive refeeding in hospitalized adolescents with anorexia nervosa result in increased hypophosphatemia? J Adolesc Health. 2010;46(6):577-82. doi:10.1016/j. jadohealth.2009.11.207.

11. Golden NH, Keane-Miller C, Sainani KL, Kapphahn CJ. Higher Caloric Intake in Hospitalized Adolescents with Anorexia Nervosa is Associated with Reduced Length of Stay and No Increased Rate of Refeeding Syndrome. J Adolesc Health. 2013;53:573-8. 
12. Madden S, Miskovic-Wheatley J, Clarke S, et al. Outcomes of rapid refeeding protocol in adolescent anorexia nervosa. J Eat Disord. 2015;3:8.

13. Garber AK, Mauldin K, Michihata N, et al. Higher calorie diets increase rate of weight gain and shorten hospital stay in hospitalized patients with anorexia nervosa. J Adolesc Health. 2013;53:579-84.

14. Leclerc A, Turrini T, Sherwood K, Katzman DK. Evaluation of nutrition rehabilitation protocol in hospitalized adolescents with restrictive eating disorder. J Adolesc Health. 2013;53:585-9.

15. Parker EK, Faruquie SS, Anderson G, et al. Higher caloric refeeding is safe for hospitalized adolescents with restrictive eating disorders. J Nutr Metab. 2016; 2016:1-9.

16. Gentile MG. Enteral nutrition for feeding severely underfed patients with anorexia nervosa. Nutrients. 2012:4(9):1293-303.

17. Gentile MG, Pastorelli P, Ciceri R, et al. Specialized refeeding treatment or anorexia nervosa patients suffering from extreme undernutrition. Clin Nutr. 2010;29:627-32.

18. Garber AK, Sawyer SM, Guarda AS, et al. A systematic review of approaches to refeeding in patients with anorexia nervosa. Int J Eat Disord. 2016;49: 293-310.

19. American Psychiatric Association. Diagnostic and statistical manual of mental disorders. 5th ed. Arlington, VA: American Psychiatric Association; 2013. ISBN 978-0-89042-554-1.

20. Le Grange D, Doyle PM, Swanson SA, et al. Calculation of expected body weight in adolescents with eating disorders. Pediatrics. 2012;129(2):e438-46.

21. Norris M. Phosphate supplementation during refeeding of hospitalized adolescents with anorexia nervosa-watch and wait of empirically treat. J Adolesc Health. 2016;58:593-4.

\section{Submit your next manuscript to BioMed Central and we will help you at every step:}

- We accept pre-submission inquiries

- Our selector tool helps you to find the most relevant journal

- We provide round the clock customer support

- Convenient online submission

- Thorough peer review

- Inclusion in PubMed and all major indexing services

- Maximum visibility for your research

Submit your manuscript at www.biomedcentral.com/submit 